\title{
Role of bicarbonate and volume therapy in the prevention of acute kidney injury in rhabdomyolysis: a retrospective propensity score-matched cohort study
}

\author{
Hye Won Kim ${ }^{1,2}$, Sejoong $\mathrm{Kim}^{3}$, Jung Hun Ohn ${ }^{1,2}$, Nak-Hyun Kim ${ }^{1,2}$, Jongchan Lee ${ }^{1,2}$, Eun Sun Kim ${ }^{1,2}$, \\ Yejee Lim $^{1,2}$, Jae Ho Cho ${ }^{1,2}$, Hee Sun Park ${ }^{1,2}$, Jiwon Ryu ${ }^{1,2}$, Sun-wook Kim ${ }^{1,2}$ \\ ${ }^{1}$ Division of General Internal Medicine, Department of Internal Medicine, Seoul National University Bundang Hospital, Seoul National \\ University College of Medicine, Seongnam, Republic of Korea \\ ${ }^{2}$ Hospital Medicine Center, Seoul National University Bundang Hospital, Seongnam, Republic of Korea \\ ${ }^{3}$ Division of Nephrology, Department of Internal Medicine, Seoul National University Bundang Hospital, Seoul National University College of \\ Medicine, Seongnam, Republic of Korea
}

Background: Although bicarbonate has traditionally been used to treat patients with rhabdomyolysis at high risk of acute kidney injury (AKI), it is unclear whether this is beneficial. This study compared bicarbonate therapy to non-bicarbonate therapy for the prevention of AKI and mortality in rhabdomyolysis patients.

Methods: In a propensity score-matched cohort study, patients with a creatine kinase (CK) level of $>1,000 \mathrm{U} / \mathrm{L}$ during hospitalization were divided into bicarbonate and non-bicarbonate groups. Patients were subgrouped based on low-volume (<3 $\mathrm{mL} / \mathrm{kg} / \mathrm{hr})$ or high-volume ( $\geq 3 \mathrm{~mL} / \mathrm{kg} / \mathrm{hr}$ ) fluid resuscitation in the first 72 hours. Logistic regression analyses were used to identify the impacts of bicarbonate use and fluid resuscitation on AKI risk and need for dialysis. The Kaplan-Meier method was used to estimate survival. Volume overload and electrolyte imbalances were assessed.

Results: Among 4,077 patients, we assembled a cohort of 887 pairs of patients treated with and without bicarbonate. Bicarbonate group had a higher incidence of AKI, higher rate of dialysis dependency, higher 30-day mortality, and longer hospital stay than the non-bicarbonate group. Further, patients who received high-volume fluid therapy had worse renal outcomes and a higher mortality than those who received low-volume fluids regardless of bicarbonate use. Bicarbonate use, volume overload, and AKI were associated with higher mortality. Volume overload was significantly higher in the bicarbonate group than in the non-bicarbonate group.

Conclusion: Bicarbonate or high-volume fluid therapy for patients with rhabdomyolysis did not reduce AKI or improve mortality compared to non-bicarbonate or low-volume fluid therapy. Limited use of bicarbonate and adjustment of fluid volume may improve the short- and long-term outcomes of patients with rhabdomyolysis.

Keywords: Acute kidney injury, Rhabdomyolysis, Sodium bicarbonate

\footnotetext{
Received: April 20, 2021; Revised: September 30, 2021; Accepted: October 10, 2021

Correspondence: Sejoong Kim

Division of Nephrology, Department of Internal Medicine, Seoul National University Bundang Hospital, 82 Gumi-ro 173beon-gil, Bundang-gu, Seongnam 13620, Republic of Korea. E-mail: sejoong@snubh.org

ORCID: https://orcid.org/0000-0002-7238-9962
}

Copyright (C) 2022 by The Korean Society of Nephrology

(a) This is an Open Access article distributed under the terms of the Creative Commons Attribution Non-Commercial and No Derivatives License (http:// creativecommons.org/licenses/by-nc-nd/4.0/) which permits unrestricted non-commercial use, distribution of the material without any modifications, and reproduction in any medium, provided the original works properly cited. 


\section{Introduction}

Acute kidney injury (AKI) occurs in $14 \%$ to $46 \%$ of patients with rhabdomyolysis [1]. When muscle cells are damaged, intracellular myoglobin is released into the circulation causing renal damage due to direct tubule injury, tubular obstruction, or renal vasoconstriction. Subsequently, a large amount of water is rapidly expelled into the extracellular fluid, causing a decreased glomerular filtration rate with worsening of AKI [2]. Life-threatening AKI leads to electrolyte and volume imbalances, which can cause arrhythmia or cardiac arrest, and renal replacement therapy is required. de Meijer et al. [3] reported that mortality in patients who developed AKI was twice as high as in those who did not. Therefore, for patients with severe rhabdomyolysis with a high risk of AKI and death, judicious and timely treatment remains essential.

The hypotheses that acidic urine worsens acute tubulonephropathy and that the risk of AKI is associated with dehydration were used to support bicarbonate therapy for patients with rhabdomyolysis, wherein bicarbonate inhibits myoglobin cast formation, and a large amount of fluid results in solute diuresis due to alkalization of the urine [4]. However, there are only a few studies, with no clear consensus, on whether the routine use of bicarbonate can prevent the development of AKI $[5,6]$. No randomized controlled clinical trials have compared bicarbonate therapy with fluid therapy alone. Indeed, some studies suggested that early, rather than late initiation of fluid therapy can help improve outcomes [7-9]; however, fluid type and target fluid volume, duration of therapy, monitoring parameters, target urine output, and the onset of initiation of fluid therapy used in these studies varied widely. Various studies have attempted to identify the optimal fluid therapy in various diseases or situations, such as renal transplant [10]. However, no prior study has investigated what fluid is optimal for the treatment of rhabdomyolysis despite the importance of fluid therapy in this condition. Thus, we investigated whether bicarbonate therapy could prevent AKI compared to fluid therapy alone and assessed the effect of fluid volume on patient outcomes. Further, we analyzed predictors of AKI, dialysis, and death in patients hospitalized for rhabdomyolysis.

\section{Methods}

\section{Study design}

We conducted a propensity score-matched cohort study in Seoul National University Bundang Hospital (a 1,334-bed, public, university-affiliated, teaching hospital). Patients with a creatine kinase (CK) level of $>1,000 \mathrm{U} / \mathrm{L}$ during hospitalization were divided into two groups; patients who received fluid with bicarbonate and those who received fluid without bicarbonate. Patients were subgrouped based on low-volume $(<3 \mathrm{~mL} / \mathrm{kg} / \mathrm{hr})$ and high-volume $(\geq 3 \mathrm{~mL} /$ $\mathrm{kg} / \mathrm{hr}$ ) fluid resuscitation in the first 72 hours of admission. Primary outcomes were the development of AKI, the incidence of dialysis, and mortality in the propensity scorematched cohort of patients exposed and unexposed to bicarbonate. The study protocol complied with the Declaration of Helsinki and received ethics approval from the Institutional Review Board of Seoul National University Bundang Hospital (No. B-1809/495-105); the need for informed consent was waived as the study did not infringe on patient privacy or health status.

\section{Study population}

We identified 6,492 hospitalized patients with a CK level of $>1,000$ U/L between March 2003 and August 2018. Based on preestablished exclusion criteria, we excluded children and adolescents under 18 years $(n=130)$ and patients with chronic kidney disease (CKD) undergoing renal replacement treatment $(n=684)$ or with cardiac enzyme elevation owing to myocardial infarction or cardiomyopathy $(\mathrm{n}=$ 1,601). Finally, 4,077 patients were included in this study. The study flow chart is presented in Fig. 1.

\section{Data collection}

All clinical records and laboratory data, including age, sex, length of hospital stay, inpatient vital signs, and laboratory values such as levels of serum $\mathrm{CK}$, blood urea nitrogen (BUN), serum creatinine ( $\mathrm{Cr}$ ), sodium, potassium, serum bicarbonate measured as total carbon dioxide $\left(\mathrm{TCO}_{2}\right)$, calcium, and albumin, were gathered from electronic medical records. Shock was defined as an initial systolic blood pressure (BP) of < $90 \mathrm{mmHg}$; additionally, a BUN/Cr ratio of $>20$ 


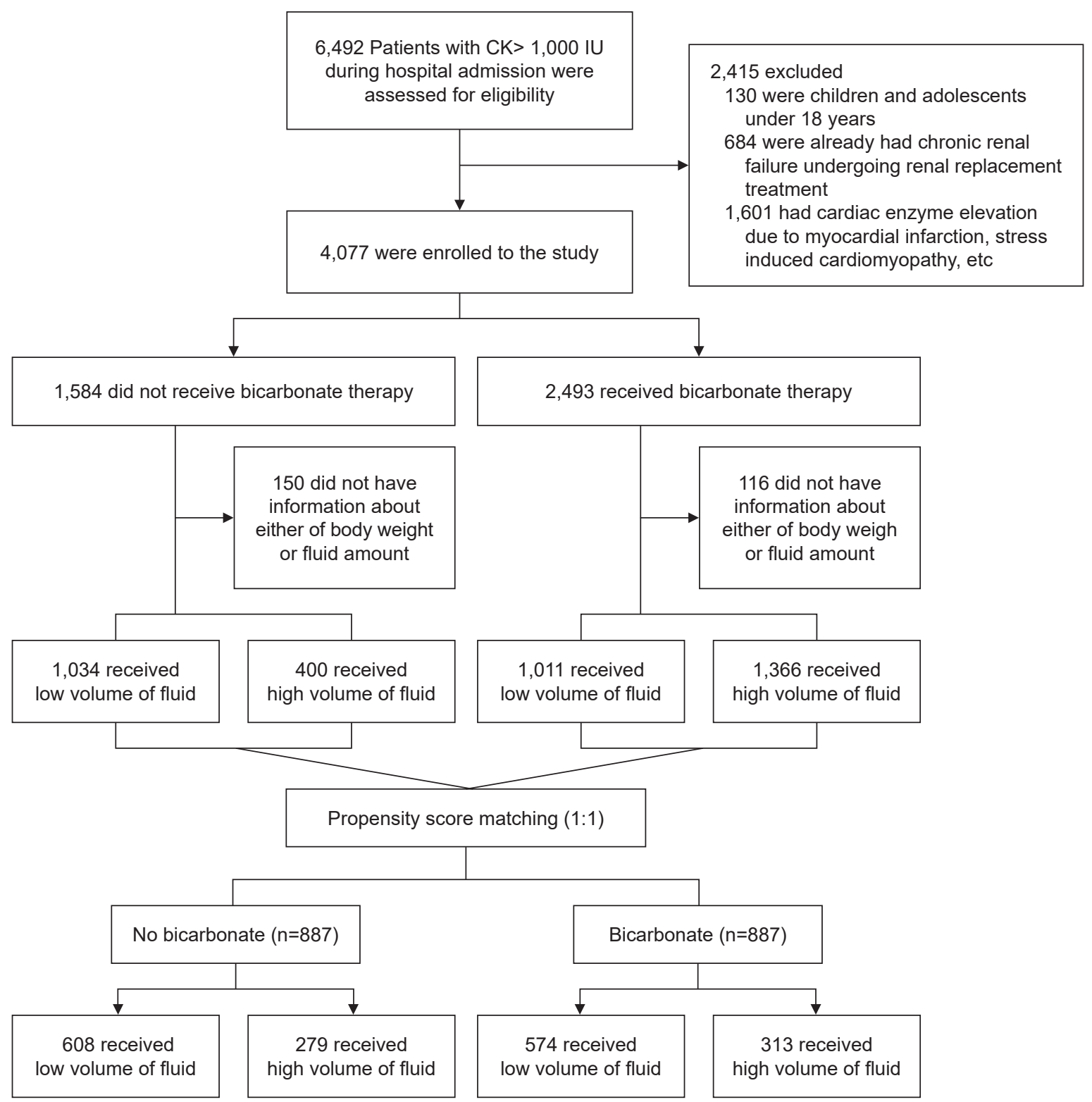

Figure 1. Study flow chart; eligible and excluded patients in the analytic data set.

CK, creatine kinase.

was used as a surrogate marker of prerenal status; that is, volume depletion. An increase of over $10 \%$ in body weight during hospitalization was regarded as volume overload. Mortality and date of death were determined from death certificates as well as the database of the Ministry of Interior and Safety of Korea.

\section{Definitions and measurements}

Onset of rhabdomyolysis was recorded as the first day of hospitalization of the patient and the occurrence of AKI was monitored throughout the hospitalization period. AKI was defined as a $\mathrm{Cr}$ level increase of $\geq 1.5$ times or $\geq 0.3 \mathrm{mg}$ / $\mathrm{dL}$ and staged according to the Clinical Practice Guideline for the Evaluation and Management of Kidney Disease: Improving Global Outcomes (KDIGO) Group. Stage 2 AKI was defined as an increase in the $\mathrm{Cr}$ to $\geq 2$ times the baseline. Stage 3 AKI was defined as a $\mathrm{Cr}$ of $\geq 3.0$ or more times the baseline, an increase in $\mathrm{Cr}$ of $\geq 4.0 \mathrm{mg} / \mathrm{dL}$, or the initiation of renal replacement therapy regardless of previous KDIGO 
stage [11]. We used the preadmission Cr value as the baseline $\mathrm{Cr}$ level. If these values were not available, we used the lowest Cr level measured during hospitalization. We confirmed cases and dates of dialysis by evaluating the Korean Society of Nephrology end-stage renal disease registry.

\section{Statistical analyses}

Baseline characteristics were analyzed as frequencies and percentages for categorical variables and as means and standard deviation (SD) for continuous variables. Unadjusted associations between the covariates and the primary outcomes were analyzed using the chi-square test for categorical data and Student $t$ test or Mann-Whitney U test for continuous data. To minimize selection bias, propensity score-matched logistic regression analysis was performed for the likelihood of bicarbonate use. A propensity score was calculated using the baseline covariates of age, sex, underlying comorbidities (diabetes, hypertension, heart failure, liver failure, and CKD), cause of rhabdomyolysis (e.g. statins), shock (systolic $\mathrm{BP}<90 \mathrm{mmHg}$ ), BUN/Cr ratio of $>20$ as a surrogate marker for volume depletion, fluid amount level, initial serum $\mathrm{TCO}_{2}$ level at admission, baseline Cr levels, and baseline CK levels (Supplementary Fig. 1, available online). The propensity score was estimated by logistic regression of the use of bicarbonate on the covariates. We used 1:1 nearest neighbor propensity score matching without replacement based on the propensity score estimated using logistic regression of the treatment on the covariates. Caliper width was set to 0.2 of the SD of the logit of the propensity score. Adequacy of balance for the covariates in the matched samples was assessed using the standardized mean difference between the two groups, with differences of $<10 \%$ reflecting good balance. Multiple logistic regression analysis models were used to identify adjusted odds ratios (ORs) for AKI and dialysis. Restricted cubic spline analysis was performed to explore the nonlinear relationship between outcomes and both serum bicarbonate levels and fluid volume. Cutoff values were calculated from maximally selected log-rank statistics using the condMC method. Comparisons between prerenal (baseline BUN/Cr ratio $>20)$ and normal (BUN/Cr ratio $\leq 20)$ groups, and between shock (systolic BP $<90 \mathrm{mmHg}$ ) and normal (systolic $\mathrm{BP} \geq$ $90 \mathrm{mmHg}$ ) groups were performed with high-volume fluid therapy and with the consequences of volume overload,
AKI, dialysis, and death within 30 days as outcomes. In addition to examining the individual effects of bicarbonate and high-volume fluid therapy on the outcome variables, further analyses were conducted to determine additional risks caused by the interaction between the use of bicarbonate and amount of fluid provided using the Relative Excess Risk due to Interaction (RERI). In brief, the RERI metric reflects the additive interaction of two risk factors. If an interaction between risk factor A and risk factor B is present, the combined effect of $A$ and $B$ is greater or smaller than the sum of the individual effects of $A$ and $B$. RERI = 0 means no interaction or exact additivity; RERI $>0$ means a positive interaction or more than additivity; and RERI $<0$ means a negative interaction. Besides RERI, interaction on an additive scale includes an attributable proportion (AP); the synergy index (S) was also computed. AP is the proportion of disease in the doubly exposed group that is attributable to the interaction and $\mathrm{S}$ is the ratio of the combined effect and the sum of the individual effects. In the absence of an additive interaction effect, AP equals 0 and S equals 1 [12]. Kaplan-Meier method was used to estimate survival and survival was compared between groups using a logrank test. A Cox proportional hazards model was used to calculate hazard ratios (HRs) for AKI and death. Logistic and Cox regression models were adjusted based on relevant baseline covariates. To estimate the significance of differences in HRs for bicarbonate use, subgroup analysis was performed using multivariate Cox proportional hazard regression, and a p-value for the interaction was estimated. R version 3.6.3 (R Foundation for Statistical Computing Platform) or IBM SPSS Statistics for Windows, version 23 (R Foundation for Statistical Computing, Vienna, Austria) were used for all analyses.

\section{Results}

\section{Participant characteristics}

Mean age of all study participants was $57.9 \pm 20.8$ years, and $66.7 \%$ of participants were male. Baseline $\mathrm{Cr}$ level was 1.0 $\pm 0.8 \mathrm{mg} / \mathrm{L}$, and $1.2 \%$ of participants had CKD as a baseline comorbidity prior to hospitalization for rhabdomyolysis. Based on medical records, we classified the cause of rhabdomyolysis as medical or surgical, with 1,834 medical (45.0\%) and 2,243 surgical cases (55.0\%). Detailed causes 
Table 1. Selected baseline characteristics and outcomes according to bicarbonate use in propensity-matched patients

\begin{tabular}{|c|c|c|c|c|c|c|}
\hline \multirow{2}{*}{ Variable } & \multicolumn{3}{|c|}{ Total population } & \multicolumn{3}{|c|}{ After propensity score matching } \\
\hline & No bicarbonate & Bicarbonate & p-value & No bicarbonate & Bicarbonate & p-value \\
\hline No. of patients & 1,584 & 2,493 & & 887 & 887 & \\
\hline Age (yr) & $61.1 \pm 17.8$ & $60.5 \pm 17.7$ & 0.23 & $61.0 \pm 17.6$ & $61.2 \pm 17.9$ & 0.79 \\
\hline Male sex & $1,077(68.0)$ & $1,644(65.9)$ & 0.19 & $612(69.0)$ & 604 (68.1) & 0.72 \\
\hline Body mass index $\left(\mathrm{kg} / \mathrm{m}^{2}\right)$ & $24.2 \pm 6.3$ & $23.9 \pm 8.7$ & 0.25 & $24.2 \pm 7.6$ & $23.8 \pm 3.9$ & 0.26 \\
\hline \multicolumn{7}{|l|}{ Comorbidity } \\
\hline Diabetes mellitus & $78(4.9)$ & $121(4.9)$ & 0.98 & $41(4.6)$ & $46(5.2)$ & 0.66 \\
\hline Hypertension & $92(5.8)$ & $150(6.0)$ & 0.84 & $55(6.2)$ & $55(6.2)$ & 0.99 \\
\hline Chronic kidney disease & $12(0.8)$ & $38(1.5)$ & 0.04 & $8(0.9)$ & $9(1.0)$ & 0.99 \\
\hline Heart failure & $0(0)$ & $3(0.1)$ & 0.43 & $0(0)$ & $0(0)$ & NA \\
\hline Liver failure & $1(0.1)$ & $20(0.8)$ & 0.003 & $1(0.1)$ & $1(0.1)$ & 0.99 \\
\hline Cause & & & & & & 0.99 \\
\hline Surgical & $869(54.9)$ & $1374(55.1)$ & 0.90 & $482(54.3)$ & $482(54.3)$ & \\
\hline Medical & $715(45.1)$ & $1,119(44.9)$ & & $405(45.7)$ & $405(45.7)$ & \\
\hline Baseline $\mathrm{Cr}(\mathrm{mg} / \mathrm{dL})$ & $0.9 \pm 0.6$ & $1.1 \pm 0.9$ & $<0.001$ & $0.9 \pm 0.7$ & $0.9 \pm 0.6$ & 0.19 \\
\hline Baseline serum CK (IU/L) & $1,330(527-2,599)$ & $1,133(287-3,194)$ & 0.02 & $1,336(553-2,577)$ & $996(206-2,572)$ & $<0.001$ \\
\hline Baseline serum $\mathrm{TCO}_{2}(\mathrm{mEq} / \mathrm{L})$ & $22.4 \pm 3.6$ & $20.7 \pm 5.0$ & $<0.001$ & $22.0 \pm 3.4$ & $21.8 \pm 4.3$ & 0.15 \\
\hline
\end{tabular}

Data are expressed as number only, mean \pm standard deviation, number $(\%)$, or median (interquartile range).

$\mathrm{CK}$, creatine kinase; $\mathrm{Cr}$, serum creatinine; NA, not applicable; $\mathrm{TCO}_{2}$, total carbon dioxide.

Table 2. Outcomes of the study participants

\begin{tabular}{|c|c|c|c|c|c|c|}
\hline \multirow{2}{*}{ Variable } & \multicolumn{3}{|c|}{ Total population } & \multicolumn{3}{|c|}{ After propensity score matching } \\
\hline & $\begin{array}{l}\text { No bicarbonate } \\
\quad(n=1,584)\end{array}$ & $\begin{array}{l}\text { Bicarbonate } \\
(n=2,493)\end{array}$ & p-value & $\begin{array}{c}\text { No bicarbonate } \\
(n=887)\end{array}$ & $\begin{array}{c}\text { Bicarbonate } \\
(\mathrm{n}=887)\end{array}$ & p-value \\
\hline \multicolumn{7}{|c|}{ Serum creatinine $(\mathrm{mg} / \mathrm{dL})$} \\
\hline Peak & $1.4 \pm 1.3$ & $2.4 \pm 2.0$ & $<0.001$ & $1.4 \pm 1.2$ & $2.1 \pm 1.7$ & $<0.001$ \\
\hline At discharge & $0.9 \pm 0.7$ & $1.3 \pm 1.2$ & $<0.001$ & $0.9 \pm 0.8$ & $1.2 \pm 1.0$ & $<0.001$ \\
\hline Follow-up & $0.9 \pm 0.7$ & $1.0 \pm 0.7$ & 0.002 & $0.9 \pm 0.7$ & $0.9 \pm 0.5$ & 0.48 \\
\hline \multicolumn{7}{|l|}{ Serum CK (IU/L) } \\
\hline Peak & $1,945(1,331-3,609)$ & $3,125(1,589-7,893)$ & $<0.001$ & $2,002(1,341-3,670)$ & $2,797(1,516-750)$ & $<0.001$ \\
\hline At discharge & $985(279-1,563)$ & $962(204-2,277)$ & 0.05 & $903(248-1,490)$ & $931(186-1,917)$ & 0.32 \\
\hline Follow-up & $77(41-151)$ & $62(33-124)$ & 0.006 & $69(38-139)$ & $62(31-126)$ & 0.16 \\
\hline \multicolumn{7}{|l|}{ Outcome } \\
\hline AKI & $730(46.1)$ & $1,974(79.2)$ & $<0.001$ & $451(50.8)$ & $671(75.6)$ & $<0.001$ \\
\hline AKI stage & & & $<0.001$ & & & $<0.001$ \\
\hline 0 & $854(53.9)$ & $519(20.8)$ & & $436(49.2)$ & $216(24.4)$ & \\
\hline 1 & $409(25.8)$ & $617(24.7)$ & & $238(26.8)$ & $240(27.1)$ & \\
\hline 2 & $175(11.0)$ & $433(17.4)$ & & $116(13.1)$ & $154(17.4)$ & \\
\hline 3 & $146(9.2)$ & 924 (37.1) & & 97 (10.9) & $277(31.2)$ & \\
\hline Dialysis & $25(1.6)$ & $473(19.0)$ & $<0.001$ & $16(1.8)$ & $129(14.5)$ & $<0.001$ \\
\hline Hospital stay (day) & $22.0 \pm 22.7$ & $26.6 \pm 54.4$ & $<0.001$ & $22.1 \pm 21.7$ & $28.1 \pm 59.6$ & 0.005 \\
\hline 30-Day mortality & $96(6.1)$ & $659(26.4)$ & $<0.001$ & $61(6.9)$ & $211(23.8)$ & $<0.001$ \\
\hline All-cause mortality & 495 (31.3) & $1,126(45.2)$ & $<0.001$ & 301 (33.9) & $404(45.5)$ & $<0.001$ \\
\hline
\end{tabular}

Data are expressed as mean \pm standard deviation, median (interquartile range), or number (\%).

$\mathrm{CK}$, creatine kinase; AKI, acute kidney injury. 
of rhabdomyolysis are described in Supplementary Table 1 (available online). Bicarbonate-containing solution was administered to 2,493 patients $(61.1 \%)$ during hospitalization. Patients from the bicarbonate group tended to be older and to have higher baseline and peak Cr levels (Table 1). Serum CK level at presentation and peak serum CK level during hospitalization were higher in the bicarbonate group than in the non-bicarbonate group.

\section{Bicarbonate use and outcomes}

A significantly higher incidence of AKI development was observed in the bicarbonate group than in the non-bicarbonate group ( $\mathrm{n}=1,974$ [79.2\%] vs. $\mathrm{n}=730$ [46.1\%], $\mathrm{p}<$ 0.001) (Table 2; Supplementary Fig. 2A, available online).
Multivariate logistic regression analysis for AKI showed that the use of bicarbonate was independently associated with increased risk of AKI development, with an OR of 3.17 (95\% confidence interval [CI], 2.62-3.85, p < 0.001).

In the propensity score-matched model, bicarbonate users still had a higher risk of AKI than the non-bicarbonate group (Supplementary Fig. 2B). Moreover, the bicarbonate group showed a statistically higher risk of dialysis dependency $(n=129$ [14.5\%] vs. $n=16$ [1.8\%], $p<0.001)$, extended hospital stay $(28.1 \pm 59.6$ days vs. $22.1 \pm 21.7$ days, $\mathrm{p}=0.005$ ) (Table 2), and higher mortality than the non-bicarbonate group (Supplementary Table 2, available online). Using multivariate logistic regression analysis, use of bicarbonate (OR, 8.97; 95\% CI, 5.05-15.91), medical cause of rhabdomyolysis (OR, 1.90; 95\% CI, 1.28-2.81), use
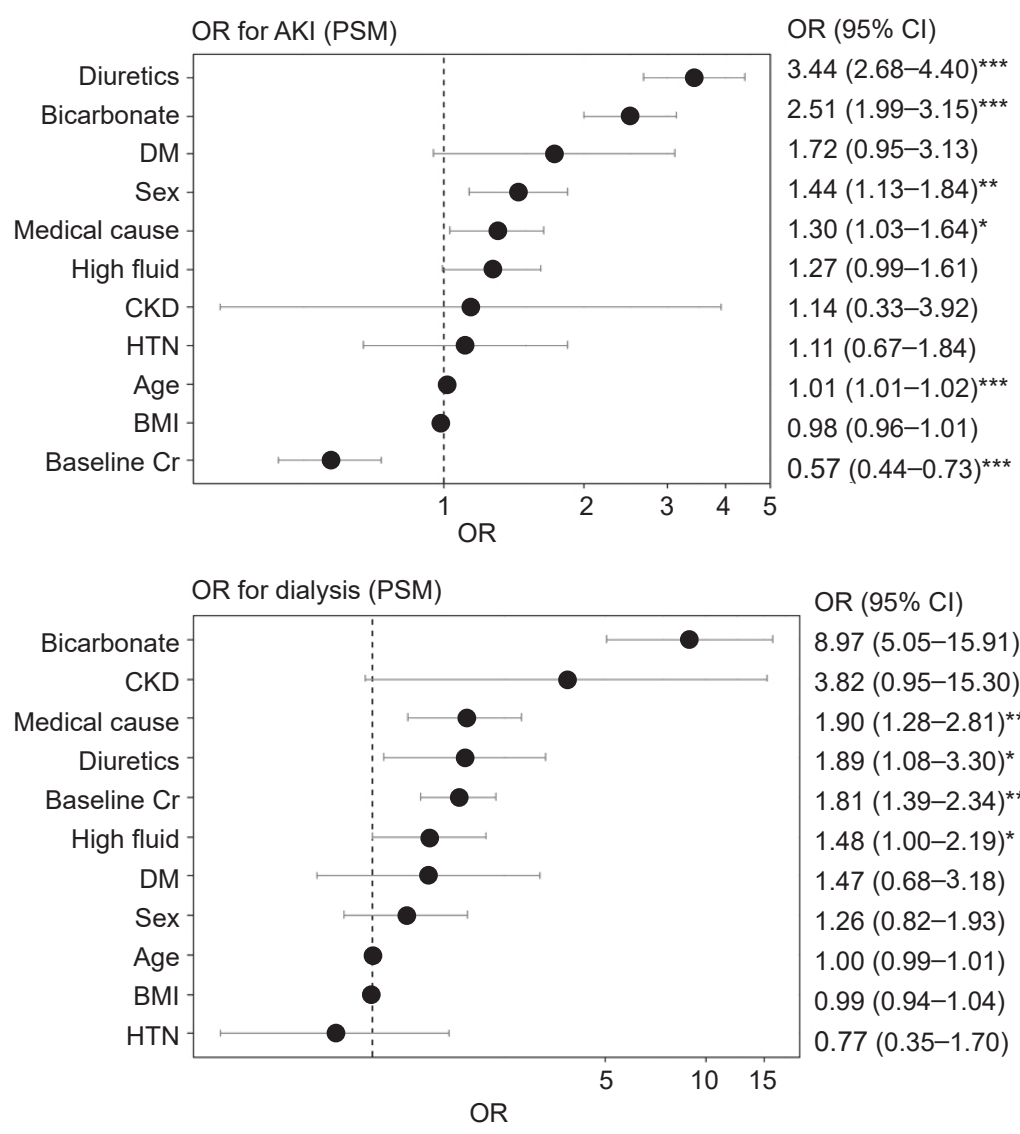

OR $(95 \% \mathrm{Cl})$

$8.97(5.05-15.91)^{* * *}$

$3.82(0.95-15.30)$

$1.90(1.28-2.81)^{* *}$

$1.89(1.08-3.30)^{*}$

$1.81(1.39-2.34)^{* * *}$

$1.48(1.00-2.19)^{*}$

$1.47(0.68-3.18)$

$1.26(0.82-1.93)$

$1.00(0.99-1.01)$

$0.99(0.94-1.04)$

$0.77(0.35-1.70)$

Figure 2. ORs for the development of AKI and the initiation of dialysis based on multivariate logistic regression of the propensity-matched cohort.

$\mathrm{AKI}$, acute kidney injury; BMI, body mass index; Cl, confidence interval; CKD, chronic kidney disease; Cr, serum creatinine; DM, diabetes mellitus; HTN, hypertension; OR, odds ratio; PSM, propensity score matching.

$* p<0.05, * * p<0.01, * * * p<0.001$. 


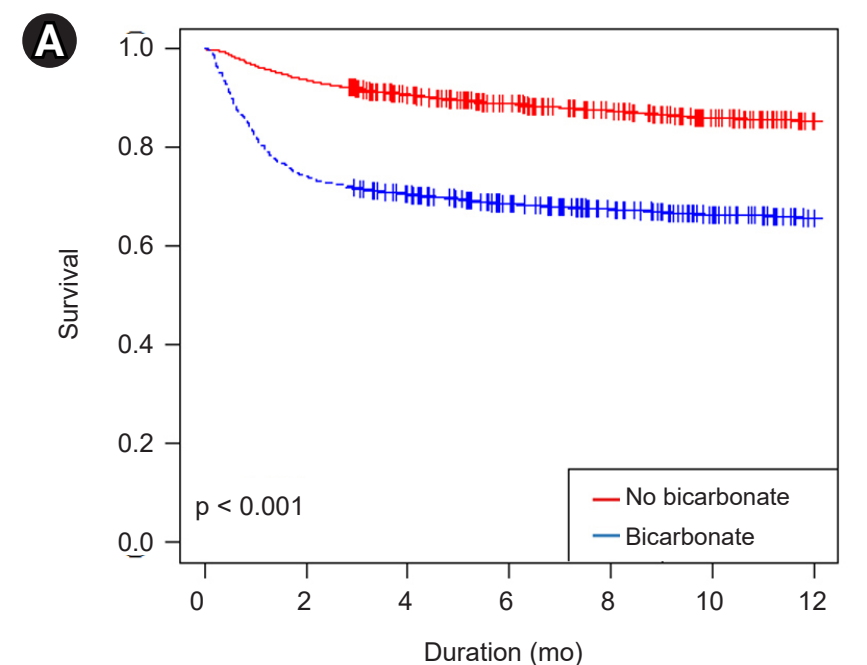

Numbers at risk

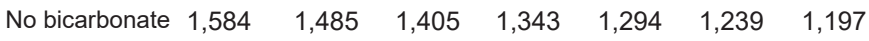

$\begin{array}{llllllll}\text { Bicarbonate } & 2,493 & 1,850 & 1,746 & 1,671 & 1,611 & 1,553 & 1,506\end{array}$
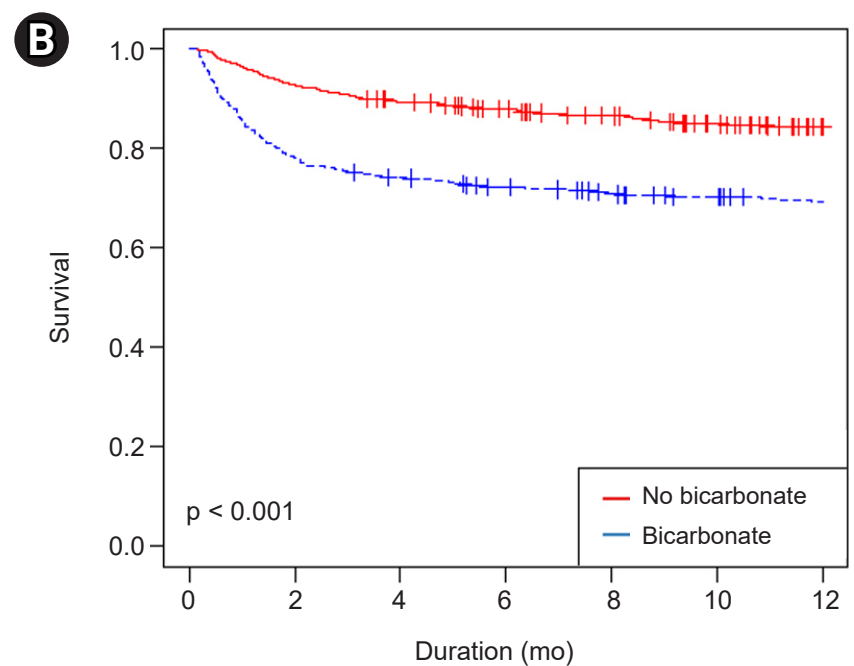

Numbers at risk

$\begin{array}{lllllll}\text { No bicarbonate } 887 & 823 & 789 & 766 & 745 & 718 & 695\end{array}$

$\begin{array}{llllllll}\text { Bicarbonate } & 887 & 693 & 655 & 633 & 616 & 604 & 591\end{array}$

Figure 3. Kaplan-Meier curves relating bicarbonate use to all-cause mortality. (A) For the total population. (B) For the propensity-matched patients.

of diuretics (OR, 1.89; 95\% CI, 1.08-3.30), higher baseline Cr (OR, 1.81; 95\% CI, 1.39-2.34), and high-volume fluid resuscitation (OR, 1.48; 95\% CI, 1.00-2.19) were independent risk factors for the need for dialysis (Fig. 2). Kaplan-Meier curves estimating the probability of all-cause mortality showed a significantly higher risk in the bicarbonate group than in the non-bicarbonate group (Fig. 3). Medical cause (adjusted HR [aHR], 1.8; 95\% CI, 1.57-2.21), volume overload (aHR, 1.47; 95\% CI, 1.18-1.82), higher AKI stage (aHR, 1.23; 95\% CI, 1.26-1.47), bicarbonate use (aHR, 1.33; 95\% CI, 1.12-1.59), and higher baseline Cr level (aHR, 1.16; 95\% CI, 1.05-1.29) were significant predictors of mortality in the multivariate Cox proportional regression analysis (Supplementary Fig. 3C, available online). Moreover, we observed that the hazards of AKI, dialysis, and mortality increased as peak serum bicarbonate, measured as the $\mathrm{TCO}_{2}$, increased to above the normal range in restricted cube splines (Supplementary Fig. 4, available online).

Differences in the hazard ratio for the development of acute kidney injury according to use of bicarbonate in prespecified strata

Subgroup analysis suggested a stronger association between bicarbonate use and AKI in patients with a surgical cause of rhabdomyolysis (OR, 1.73; 95\% CI, 1.50-1.73) than in patients with a medical cause (OR, 1.56; 95\% CI, 1.351.81) (p for interaction $<0.001$ ), although bicarbonate use was significantly associated with AKI in patients with either medically or surgically-induced rhabdomyolysis (Fig. 4). Irrespective of age, sex, underlying comorbidities except for $\mathrm{CKD}$, fluid amount, baseline serum $\mathrm{TCO}_{2}, \mathrm{BUN} / \mathrm{Cr}$ ratio, or $\mathrm{BP}$, the use of bicarbonate was associated with AKI across subgroups of patients (Fig. 4).

\section{Fluid resuscitation and outcomes}

Slightly more patients received low-volume fluid resuscitation than high-volume fluid resuscitation $(53.7 \%$ and $46.3 \%$, respectively). Patients administered a high volume of fluid had a higher incidence of AKI regardless of the use of bicarbonate (AKI: OR, 2.09; 95\% CI, 1.82-2.41; p < 0.001; dialysis: OR, 2.05; 95\% CI, 1.68-2.49; $\mathrm{p}<0.001$; AKI in the propensity score-matched cohort: OR, 1.40; 95\% CI, 1.14-1.73; $\mathrm{p}=0.001$; and dialysis in the propensity scorematched cohort: OR, 1.28; 95\% CI, 0.90-1.82; $\mathrm{p}=0.163$ ) (Fig. 5). Hazards of AKI, dialysis, and mortality increased as the amount of fluid received increased during the first 72 hours of treatment (Supplementary Fig. 4). According to the estimated cutoff value calculated using the maximally 


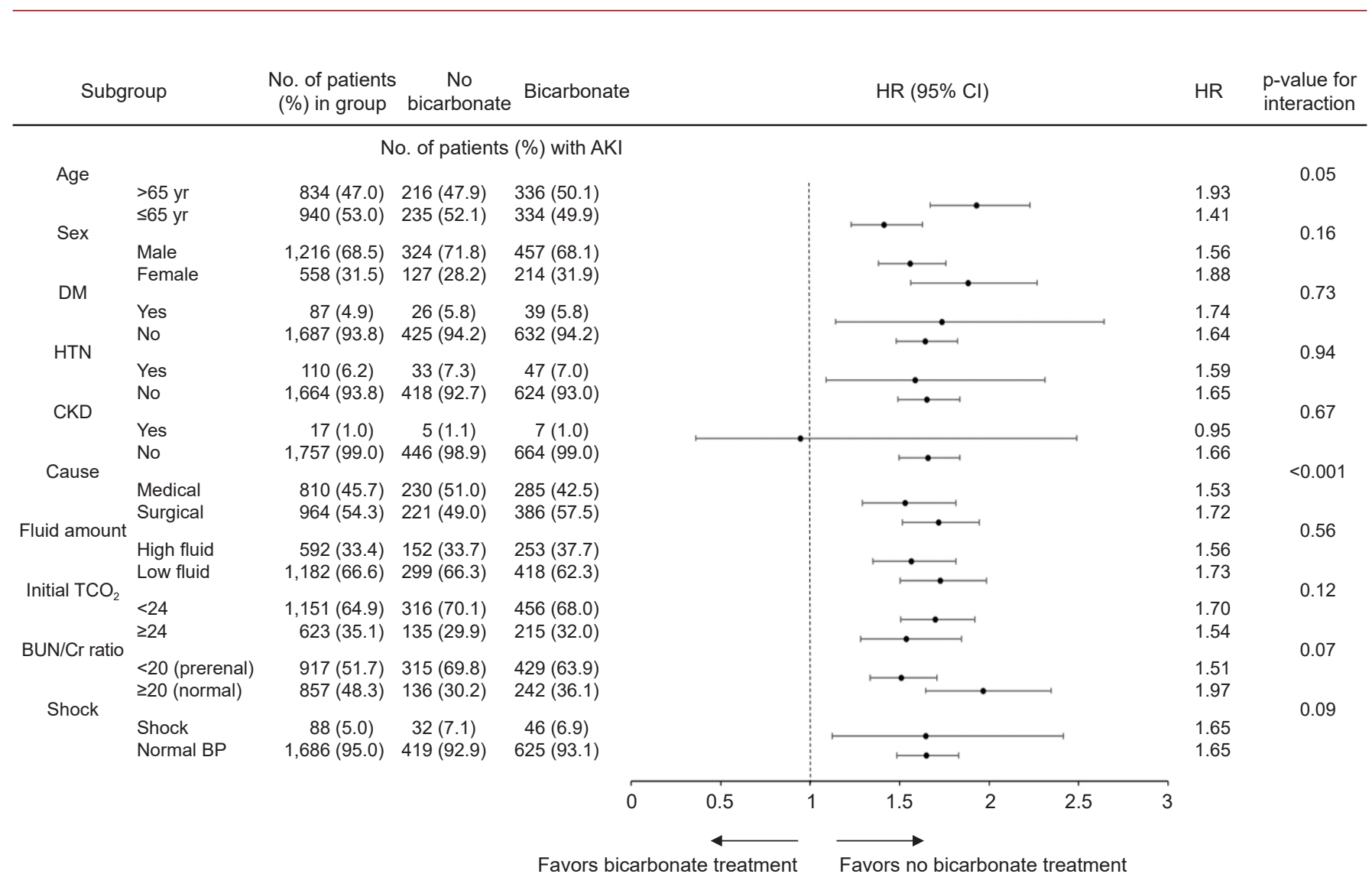

Figure 4. Differences in HRs for the development of AKI with respect to the use of bicarbonate in prespecified strata.

AKI, acute kidney injury; BP, blood pressure; BUN/Cr, blood urea nitrogen to creatinine; CKD, chronic kidney disease; DM, diabetes mellitus; HTN, hypertension; HR, hazard ratio; $\mathrm{TCO}_{2}$, total carbon dioxide.
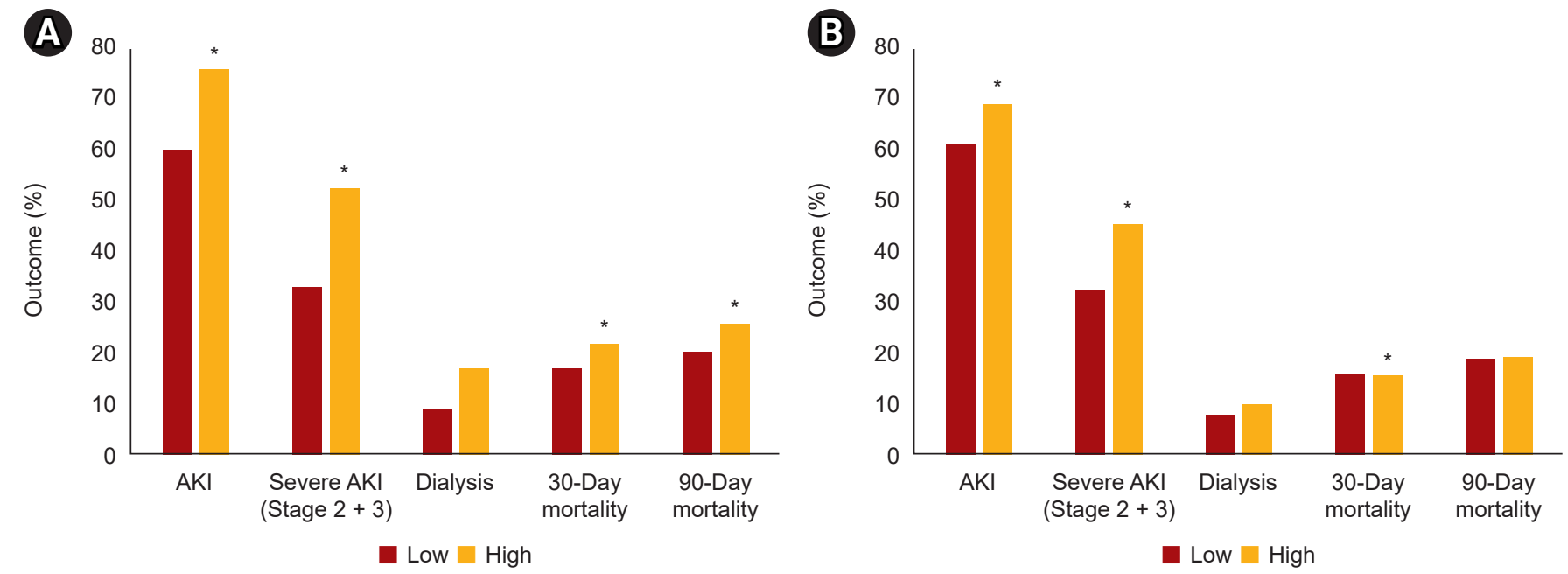

Figure 5. Rate of AKI, severe AKI, dialysis, and mortality according to the amount of fluid treatment for rhabdomyolysis. (A) Total population. (B) After propensity score matching. AKI, acute kidney injury. 
Table 3. Analysis of the effects of interaction between BIC therapy and high-volume fluid treatment on the development of acute kidney injury

\begin{tabular}{|c|c|c|c|c|c|}
\hline \multirow{2}{*}{ Variable } & \multicolumn{2}{|c|}{ No bicarbonate } & \multicolumn{2}{|c|}{ Bicarbonate } & \multirow{2}{*}{$\begin{array}{c}\text { OR for bicarbonate group } \\
\text { within strata of the fluid group }\end{array}$} \\
\hline & No. of patients ${ }^{a}$ & OR (95\% Cl) & No. of patients ${ }^{a}$ & OR $(95 \% \mathrm{Cl})$ & \\
\hline Low-volume fluid & 299/309 & 1.0 (reference) & $418 / 156$ & $1.62(1.39-1.87)$ & 1.62 \\
\hline High-volume fluid & $152 / 127$ & $1.14(0.93-1.38)$ & $253 / 60$ & $1.81(1.53-2.14)$ & 1.59 \\
\hline $\begin{array}{l}\text { OR for the fluid group within } \\
\text { strata of the bicarbonate group }\end{array}$ & & 1.14 & & 1.12 & \\
\hline
\end{tabular}

Measure of interaction on additive scale $(95 \% \mathrm{Cl})$ : relative excess risk due to interaction, $1.56(0.86-2.27)$; attributable proportion due to interaction, 0.56 (0.42-0.71); and synergistic index, 8.65 (2.02-37.04).

$\mathrm{Cl}$, confidence interval; OR, odds ratio.

${ }^{a}$ With/without acute kidney injury.

selected log-rank statistics with the condMC method, over $5.5 \mathrm{~mL} / \mathrm{kg} / \mathrm{hr}$ of fluid for a period of 72 hours was associated with higher mortality $(\mathrm{p}<0.001)$. The low-volume fluid group had a lower risk of mortality in Kaplan-Meier analysis, and the cutoff fluid rate of $5.5 \mathrm{~mL} / \mathrm{kg} / \mathrm{hr}$ maximized the difference in survival estimates (Supplementary Fig. 5, available online).

As expected, patients in the prerenal (baseline BUN/ Cr ratio $>20$ ) and shock (systolic $\mathrm{BP}<90 \mathrm{mmHg}$ ) groups received high-volume fluid therapy and had higher rates of volume overload, AKI, dialysis, and death within 30 days. In subgroup analyses stratified by both BP status (normal BP vs. shock) and volume status (BUN/Cr ratio of $\leq 20$ vs. $>20$ ), high-volume fluid therapy was associated with a significantly higher rate of AKI, dialysis, severe AKI, and death in 30 days in every subgroup except the shock group, indicating that high-volume fluid therapy itself has a negative effect on prognosis (Supplementary Table 3, 4; available online).

Interaction between use of bicarbonate and fluid amount

Given that the use of both bicarbonate and high-volume fluid therapies were associated with a poor prognosis, we evaluated additive risks using the RERI and found a significant additive interaction between bicarbonate and high-volume treatment for the development of AKI in the propensity score-matched cohort using a multi-adjusted model (Table 3). The RERI was 1.56 , suggesting a 1.56 relative excess risk owing to the additive interaction. We also found that $56 \%$ of the OR for developing AKI in patients exposed to both bicarbonate and high-volume fluid therapy was attributable to the additive interaction between bicar- bonate and high fluid volume. Moreover, the risk of AKI in bicarbonate users who received high-volume fluid therapy was 8.65 times as high as the sum of the risks in participants exposed to a single risk factor alone. This indicates that bicarbonate and high-volume fluid treatments interact synergistically to cause AKI.

\section{Volume overload and electrolyte imbalances}

Patients who received bicarbonate treatment developed volume overload more frequently than patients who did $\operatorname{not}(\mathrm{n}=418[16.8 \%]$ vs. $\mathrm{n}=126$ [8.0\%], $\mathrm{p}<0.001)$ (Supplementary Fig. 6, available online). Similarly, more patients in the high- volume fluid group developed volume overload than those in the low-volume fluid group ( $\mathrm{n}=195$ [9.5\%] vs. $\mathrm{n}=321$ [18.2\%], $\mathrm{p}<0.001)$. Electrolyte imbalances such as hyponatremia, hypernatremia, hypokalemia, hyperkalemia, hypochloremia, and hyperchloremia were more common in the bicarbonate group and high-volume fluid group than the non-bicarbonate group and low-volume fluid group.

\section{Discussion}

In this retrospective, propensity score-matched cohort study involving patients with rhabdomyolysis, we concluded that bicarbonate therapy increased AKI risk, the need for dialysis, and mortality. Additional hypervolemic treatment also appeared to be deleterious as it was associated with volume overload and resulted in poor renal outcomes.

Early fluid resuscitation and bicarbonate therapy had been advocated since a 1984 study of seven participants with crush injuries reported treatment by the induction of 
alkaline solute diuresis [13]; since then, however, results concerning the outcomes of bicarbonate treatment for the prevention of AKI have been conflicting [5,14-17]. In a previous retrospective study of more than 2,000 patients with traumatic rhabdomyolysis, bicarbonate with mannitol did not prevent AKI, the need for dialysis, or decrease mortality. Thus, the authors concluded that the use of bicarbonate with mannitol should be reevaluated [6]. Several meta-analyses have noted the lack of prospective controlled trials comparing bicarbonate therapy with fluid therapy alone for preventing rhabdomyolysis-induced AKI $[8,9]$.

In our study, higher rates of AKI in the bicarbonate group might have resulted from several factors. First, clinicians may have used bicarbonate therapy $a$ priori in patients with presumptive high-risk factors with worse expected disease courses because its use was at the discretion of the treating physicians. We used propensity score matching by adjusting for underlying disease, sex, volume and BP status, baseline $\mathrm{Cr}$ level, and baseline CK level in an attempt to correct for this; however, the bicarbonate group still presented with a higher AKI incidence, need for dialysis, and mortality. Based on a review of the literature for other studies with higher AKI rates in the bicarbonate group than in the non-bicarbonate group, we found one study that reported that of 157 patients, $16.5 \%$ had AKI and the incidence of AKI was significantly higher in those patients who received bicarbonate therapy; however, the authors speculated that bicarbonate administration was not the cause of AKI but the result of receiving more aggressive therapy [18]. However, this study was not designed to identify the difference between bicarbonate and non-bicarbonate groups; thus, causal relations could not be assessed. Second, bicarbonate therapy may potentially have detrimental effects. The effects of volume and sodium overload are not disputable [19]. Consequences of bicarbonate therapy are hypervolemia, hyperosmolarity, and hypernatremia. Bicarbonate infusion may induce hyperlactatemia. In contrast to the beneficial use of bicarbonate for bicarbonate-losing metabolic acidosis, such as metabolic acidosis caused by diarrhea or renal tubular acidosis, the use of bicarbonate for lactic acidosis has been disproved [19]. Furthermore, a hyperosmolar state caused by bicarbonate infusion may lead to paradoxical intracellular acidosis $[8,20]$. Additionally, serum ionized calcium level is reduced by bicarbonate infusion, and hypocalcemia is associated with reduced left ventricular contractility [21]. These reactions may offset any beneficial effect of alkalization of urine, and bicarbonate therapy may contribute to the development of AKI. We found that hypernatremia, volume overload, and other electrolyte imbalances were more prominent in the bicarbonate group than in the non-bicarbonate group, although the rates of hypocalcemia were not significantly different between the bicarbonate and non-bicarbonate groups, and lactate levels were not measured.

Hypervolemic treatment may also contribute to the development of AKI [2]. The primary mechanism of rhabdomyolysis-induced AKI is renal vasoconstriction; thus, early intravenous fluid treatment has been used to restore blood flow and glomerular filtration to protect the kidneys. Surprisingly, previous studies have shown that a greater degree of kidney dysfunction results from venous congestion transmitted to the renal venous compartment than from lack of arterial perfusion $[22,23]$. Therefore, aggressive volume treatment to maintain renal flow may contribute to peripheral overload, resulting in renal congestion and AKI. We demonstrated that patients with volume overload were at increased risk of developing AKI. Moreover, volume overload was an independent risk factor for mortality in our study. Our results are consistent with those of a previous study that reported that volume overload increases the risk of AKI and mortality, independent of the severity of acute illness, indicating that prevention of volume overload is crucial in managing critically ill patients [24]. We stratified high-volume fluid therapy and bicarbonate therapy using RERI analysis, and each variable independently and synergistically had detrimental effects on the development of AKI. We suggest that $<5.5 \mathrm{~mL} / \mathrm{kg} / \mathrm{hr}$ fluid resuscitation should be used to prevent AKI and mortality in patients with rhabdomyolysis, and volume overload, hourly urine output, and central venous pressure should be monitored periodically. Nevertheless, bicarbonate use may have a role in severe acidemic patients with rhabdomyolysis. Despite the lack of relevant clinical data supporting its effectiveness, theoretical evidence still exists [25-27].

Although the risk of AKI was higher for medically-caused rhabdomyolysis than surgically-induced rhabdomyolysis, the association between bicarbonate use and AKI was higher in patients with rhabdomyolysis with a surgical cause. In terms of AKI, bicarbonate use appeared harmful in patients who were postsurgical, hypothermic, or im- 
mobilized, whereas bicarbonate use appeared beneficial in cases of crush, seizure, hyperthermia, and malignant neuroleptic syndrome. However, given the large numerical differences in causal subcategories in our cohort, further subanalyses are required to elucidate important subgroup effects. Therefore, we propose a large-scale cohort study wherein patients have rhabdomyolysis of homogeneous etiology.

Our study had several strengths. We used propensity score-matched analyses to reduce selection bias and potential baseline differences between bicarbonate and non-bicarbonate groups. AKI was defined and staged according to standard definitions proposed by the KDIGO Group [11]; therefore, the study results are likely generalizable to patients at other institutions. More than 4,000 patients were enrolled, and after propensity score matching, over 3,000 patients were included in the analyses, which to the best of our knowledge is the largest cohort of rhabdomyolysis patients analyzed in a single study.

Our study also has several limitations. First, it was not specifically designed to illustrate the initiation and maintenance of bicarbonate therapy, bicarbonate dosage, or target serum and urine $\mathrm{pH}$ levels. We did not tailor the infusion of bicarbonate according to serum or urine $\mathrm{pH}$ levels. Second, information on the type of fluid was not stratified. Different fluid mixtures such as isotonic saline and dextrose fluids were used in our study in the real-world setting. However, the amount of fluid was adjusted by body weight to set individual target volumes. Third, although we separated medical and surgical causes of rhabdomyolysis, numerous heterogeneous causes of rhabdomyolysis were included. Fourth, although we performed propensity score matching to avoid potential bias in the use of bicarbonate in patients with predicted worse outcomes, such as severe acidemia, volume depletion, or state of shock, there may have been confounding effects due to complex and unintended treatment settings. Future research should prospectively evaluate the need for bicarbonate infusion and the amount of fluid therapy without these limitations.

In conclusion, we found that the use of bicarbonate and high-volume fluid treatment that resulted in volume overload were not beneficial but rather harmful to a certain subset of patients with rhabdomyolysis, as reflected by increased AKI risk, the need for dialysis, and increased mortality.

\section{Conflicts of interest}

All authors have no conflicts of interest to declare.

\section{Funding}

This work was supported by a grant from the Seoul National University Bundang Hospital Research Fund (grant no. 02-2019-034).

\section{Acknowledgments}

We would like to acknowledge So Yeon Ahn, Associate Professor at the Department of Medical Research Collaborating Center, Seoul National University Bundang Hospital, for statistical counseling. We thank Yunseo Lee, a student at Seoul National University Medical School, for his assistance in data acquisition and cleaning.

\section{Authors' contributions}

Conceptualization, Project administration, Supervision: SK Data curation, Formal analysis, Visualization: HWK

Funding acquisition: HWK, SK, YL, JHO

Investigation, Software: HWK, SK, JHO, NHK

Methodology: HWK, SK

Resources: HWK, SK, JL, ESK, YL, JHC, HSP, JR, SK

Writing-original draft: $\mathrm{HWK}$

Writing-review \& editing: HWK, SK, JL, ESK, YL, JHC, HSP, JR, SK

All authors read and approved the final manuscript.

\section{ORCID}

Hye Won Kim, https://orcid.org/0000-0001-9450-3626 Sejoong Kim, https://orcid.org/0000-0002-7238-9962 Jung Hun Ohn, https://orcid.org/0000-0001-5415-4505 Nak-Hyun Kim, https://orcid.org/0000-0003-1134-1364 Jongchan Lee, https://orcid.org/0000-0001-6590-2353 Eun Sun Kim, https://orcid.org/0000-0002-6024-5777 Yejee Lim, https://orcid.org/0000-0002-3540-0202 Jae Ho Cho, https://orcid.org/0000-0002-8391-0557 Hee Sun Park, https://orcid.org/0000-0003-4408-507X Jiwon Ryu, https://orcid.org/0000-0002-2372-8948 Sun-wook Kim, https://orcid.org/0000-0003-1506-7366 


\section{References}

1. Zutt R, van der Kooi AJ, Linthorst GE, Wanders RJ, de Visser M. Rhabdomyolysis: review of the literature. Neuromuscul Disord 2014;24:651-659.

2. Boutaud O, Roberts LJ 2nd. Mechanism-based therapeutic approaches to rhabdomyolysis-induced renal failure. Free Radic Biol Med 2011;51:1062-1067.

3. de Meijer AR, Fikkers BG, de Keijzer MH, van Engelen BG, Drenth JP. Serum creatine kinase as predictor of clinical course in rhabdomyolysis: a 5-year intensive care survey. Intensive Care Med 2003;29:1121-1125.

4. Zager RA, Gamelin LM. Pathogenetic mechanisms in experimental hemoglobinuric acute renal failure. Am J Physiol 1989;256(3 Pt 2):F446-F455.

5. Homsi E, Barreiro MF, Orlando JM, Higa EM. Prophylaxis of acute renal failure in patients with rhabdomyolysis. Ren Fail 1997;19:283-288.

6. Brown CV, Rhee P, Chan L, Evans K, Demetriades D, Velmahos GC. Preventing renal failure in patients with rhabdomyolysis: do bicarbonate and mannitol make a difference? J Trauma 2004;56:1191-1196.

7. Michelsen J, Cordtz J, Liboriussen L, et al. Prevention of rhabdomyolysis-induced acute kidney injury: a DASAIM/DSIT clinical practice guideline. Acta Anaesthesiol Scand 2019;63:576-586.

8. Chavez LO, Leon M, Einav S, Varon J. Beyond muscle destruction: a systematic review of rhabdomyolysis for clinical practice. Crit Care 2016;20:135.

9. Scharman EJ, Troutman WG. Prevention of kidney injury following rhabdomyolysis: a systematic review. Ann Pharmacother 2013;47:90-105.

10. Jahangir A, Sahra S, Niazi MR, et al. Comparison of normal saline solution with low-chloride solutions in renal transplants: a meta-analysis. Kidney Res Clin Pract 2021;40:484-495.

11. Kellum JA, Lameire N; KDIGO AKI Guideline Work Group. Diagnosis, evaluation, and management of acute kidney injury: a KDIGO summary (Part 1). Crit Care 2013;17:204.

12. Rothman KJ, Greenland S, Walker AM. Concepts of interaction. Am J Epidemiol 1980;112:467-470.

13. Ron D, Taitelman U, Michaelson M, Bar-Joseph G, Bursztein S, Better OS. Prevention of acute renal failure in traumatic rhabdo- myolysis. Arch Intern Med 1984;144:277-280.

14. Mannix R, Tan ML, Wright R, Baskin M. Acute pediatric rhabdomyolysis: causes and rates of renal failure. Pediatrics 2006;118:2119-2125.

15. Altintepe L, Guney I, Tonbul Z, et al. Early and intensive fluid replacement prevents acute renal failure in the crush cases associated with spontaneous collapse of an apartment in Konya. Ren Fail 2007;29:737-741.

16. Zepeda-Orozco D, Ault BH, Jones DP. Factors associated with acute renal failure in children with rhabdomyolysis. Pediatr Nephrol 2008;23:2281-2284.

17. Nielsen JS, Sally M, Mullins RJ, et al. Bicarbonate and mannitol treatment for traumatic rhabdomyolysis revisited. Am J Surg 2017;213:73-79.

18. Ward MM. Factors predictive of acute renal failure in rhabdomyolysis. Arch Intern Med 1988;148:1553-1557.

19. Forsythe SM, Schmidt GA. Sodium bicarbonate for the treatment of lactic acidosis. Chest 2000;117:260-267.

20. Somagutta MR, Pagad S, Sridharan S, et al. Role of bicarbonates and mannitol in rhabdomyolysis: a comprehensive review. $\mathrm{Cu}$ reus 2020;12:e9742.

21. Lang RM, Fellner SK, Neumann A, Bushinsky DA, Borow KM. Left ventricular contractility varies directly with blood ionized calcium. Ann Intern Med 1988;108:524-529.

22. Ding X, Cheng Z, Qian Q. Intravenous fluids and acute kidney injury. Blood Purif 2017;43:163-172.

23. Chen X, Wang X, Honore PM, Spapen HD, Liu D. Renal failure in critically ill patients, beware of applying (central venous) pressure on the kidney. Ann Intensive Care 2018;8:91.

24. Medina-Liabres KR, Jeong JC, Oh HJ, et al. Mortality predictors in critically ill patients with acute kidney injury requiring continuous renal replacement therapy. Kidney Res Clin Pract 2021;40:401-410.

25. Bosch X, Poch E, Grau JM. Rhabdomyolysis and acute kidney injury. N Engl J Med 2009;361:62-72.

26. Zager RA. Rhabdomyolysis and myohemoglobinuric acute renal failure. Kidney Int 1996;49:314-326.

27. Cervellin G, Comelli I, Lippi G. Rhabdomyolysis: historical background, clinical, diagnostic and therapeutic features. Clin Chem Lab Med 2010;48:749-756. 